\title{
Irisin and Carcinoembryonic Antigen (CEA) as Potential Diagnostic Biomarkers in Gastric and Colorectal Cancers
}

\author{
Ahmed Abd Temur ${ }^{1}$ and Farah Aqeel Rashid*2
}

\begin{abstract}
Background: Carcinoembryonic antigen (CEA) is a common gastrointestinal tumor biomarker. Irisin is adipo-myokines that has been suggested to have a potential role in cancer development. However, limited studies test irisin as biomarker in gastric and colorectal cancers. Therefore, this study aims to investigate whether CEA and irisin could be a potential diagnostic biomarker in gastric and colorectal cancer.

Methods: A case-control study consists of 90 subjects, 21 gastric cancer patients, 49 colorectal cancer patients and 20 control. Serum CEA was detected by fluorescence immunoassay (FIA) kit. Serum irisin was determined by enzyme-linked immunosorbent assay (ELISA) kit.

Results: Serum CEA increases significantly and serum irisin decreases significantly in gastric and colorectal cancer patients. According to Receiver Operating Characteristic (ROC) curve analysis, in gastric cancer, the area under curve of CEA is 1.00 (95\% CI, 1.000-1.000, p< 0.0001). The diagnostic cut-off of CEA is $<3.08 \mathrm{ng} / \mathrm{ml}$ with $\% 100$ sensitivity and $100 \%$ specificity. The area under curve of irisin is 0.94 (95\% CI, 0.8177-1.000, $\mathrm{p}<0.0001)$. The cut-off of irisin is $>30.2 \mathrm{ng} / \mathrm{ml}$ with $\% 90$ sensitivity and $100 \%$, specificity. In colorectal cancer, the area under curve of CEA is 0.99 (95\% CI, 0.9866-1.000, $\mathrm{p}<$ 0.0001 ) and the diagnostic value $<2.6 \mathrm{ng} / \mathrm{ml}$ with $\% 98$ sensitivity and $\% 100$ specificity. The area under curve of irisin is 0.96 (95\% CI, 0.9155-1.000, $\mathrm{p}<0.0001)$. The diagnostic cut-off of irisin is $>41.9 \mathrm{ng} / \mathrm{ml}$ with 88.1 sensitivity and 90.5 specificity.
\end{abstract}

Conclusions: CEA and irisin could be powerful potential diagnostic biomarkers which would be use for early detection of gastric and colorectal cancers.

Keywords: Biomarker, Carcinoembryonic antigen (CEA), Colorectal cancer, Gastric cancer, Irisin.

\section{Introduction}

In gastrointestinal system, gastric and colorectal cancers are the most cancer -related deaths. According to global cancer statistic 2020, gastric cancer incidence ranked fifth and it is the fourth cancer-related death. Colorectal cancer incidence occupied third while it is second cancer-related death (1). The most significant issue of these two cancers is once the diseases diagnose, cancer cells spread to another organ and metastasis formed. Recently, many researchers have been attracted attention for early diagnosis which could be help in choosing effective treatment and cancer management. Therefore, investigation a biomarker for early detection is urgently needed.

The most common biomarker for gastric and colorectal cancers is carcinoembryonic antigen (CEA). CEA is a member immunoglobulin superfamily and is used as tumor marker for detection gastrointestinal cancer. In addition, it has been shown in many studies that CEA is associated with different type of cancers. In breast cancer, it was reported that CEA is an independent prognostic factor for disease free 
survival and overall survival(2, 3). In rectal cancer, it was reported that elevated CEA level is an indicator for prognosis disease recurrence and survival rate $(4,5)$. In term of diagnosis, it was reported in meta-analysis that CEA could be use as diagnosis for gastric cancer in combination with different tumor biomarkers (6).

Irisin is a dipo-myokines hormone that forms as a result of proteolytically cleavage of the fibronectin type III domain containing protein 5 (FNDC5) which encoded exercise-induce gene (7-9). Irisin is associated with obesity, diabetes mellitus, insulin resistance, chronic disease and other metabolic diseases (10-16). The function of irisin relates to energy hemostatis by browning white adipose tissue and produce heat (7). However, there is limited data about function of irisin in cancer. Recently, there are substantial evidence suggest that irisin have a potential role in cancer development (17). It was found that irisin express at high level in gastrointestinal cancers tissues (18). On the other hand, some studies suggested that irisin have an anti-cancer role. For instance, in lung cancer, it was revealed that irisin have an inhibitory effect on epithelial-mesenchymal transition and decrease invasion of cancer cells (19). In addition, it was reported that irisin significantly inhibits the growth of pancreatic cancer cell lines (20). Interestingly, irisin has been investigated as a biomarker in many malignancies. In prostate and breast cancer, it was found that reduced irisin level in prostate cancer patients in compared to healthy control. Furthermore, irisin has ability to discriminate cancer patients from controls, significantly (21, 22). Moreover, serum irisin level is higher in renal cancer patients than in control group as well as irisin has a diagnostic value, suggesting the potential application of irisin as biomarker (23). However, there are limited studies prove the possibility to use irisin as biomarker in gastric and colorectal cancer. Therefore, the aim of this study is to investigate the differences of CEA and irisin level in gastric and colorectal cancer in comparison with healthy control and then investigate whether or not CEA and irisin could be a diagnostic biomarker in gastric and colorectal cancer.

\section{Materials and Methods Study design}

case-control study consists of 90 subjects, 21 gastric cancer patients, 49 colorectal (31 colon and 18 rectal) cancer patients, and 20 healthy individuals. This study was recruited at Oncology Teaching hospital in Baghdad from $15^{\text {th }}$ November 2020 to $25^{\text {th }}$ April 2021. Patient's age ranged between (30-85) years old. Ethical approval (999) was obtained from committee in the Ministry of Health in Baghdad, Iraq. Before blood collection, the agreements were obtained for all patients and controls. All the patients were newly diagnosed by endoscopic biopsy after overnight fasting. The cancer patients and healthy individuals were matched in term of Body Mass Index (BMI). Patients, who are alcohol drink, smoking, doing regular physical exercises and who have diabetes, cardiovascular disease, hyper- or hypotension, were excluded from study.

\section{Blood analysis and biochemical measurements} peripheral venous blood $(5 \mathrm{ml})$ was collected in gel tube (MEDMAY, China) from preoperative patient and from healthy control after overnight fasting. The serum was separated after centrifugation at $1200 \mathrm{rpm}$ for $10 \mathrm{~min}$. Serum was stored at $-20^{\circ} \mathrm{C}$ until. CEA concentration was detected using fluorescence immunoassay (FIA) kit (Boditech Med Inc. Korea). Irisin concentration was determined using sandwich enzyme-linked immunosorbed assay (ELISA) kit (Mybiosource, USA). The coefficient variations of irisin kit are $<80 \%$ for intra-assay and $<10 \%$ for inter-assay.

\section{Statistical analysis}

Normal distribution of variables was assessed by Kolmogorov-Smirnov test. Normal distributed variables are expressed as mean \pm SEM and skew distributed variables are expressed as median with $25^{\text {th }}-75^{\text {th }}$ percentiles ranges. Differences between cancer patients and 
controls were analyzed by unpaired two-tailed t-test and Mann-whiteny $U$ test for normal distributed variables and for skew distributed variables, respectively. Receiver operating characteristic (ROC) curve was preformed to determine the optimal diagnostic value of irisin and CEA. The statistical analysis was performed by GraphPad Prism (8.0.2). Statistically significant was considered if the probability value less than 0.05 .

\section{Results}

Irisin and CEA levels were investigated in gastric cancer and colorectal cancer, as it was shown in table 1 . There is a strong significant decrease $(p<0.0001)$ in irisin level in gastric and in colorectal cancer patients in comparison with control group $(33.19 \pm 11.77,31.97$ (49.34.4) vs $56.01 \pm 10.95, \quad 55.9$ (43.9-76.3)), respectively. CEA level is significantly higher $(p<0.0001)$ in gastric and colorectal cancer patients than in control (6.38(113.6-3.87), 6.63(216.97 -1.66) vs 1.25(1-2.3), 1.25(1$2.3)$ ), respectively.

In order to test the potential role of irisin in diagnostic gastric and colorectal cancer patients from healthy control, receiver operating characteristic curve was preformed, as it was shown in Table 2 and Figure1. In both gastric cancer and colorectal cancer, it was observed that CEA is the most effective diagnostic tool. However, irisin still have ability to discriminate gastric and colorectal cancer from healthy individual.

Table 1. Irisin and CEA level in gastric and colorectal cancers.

\begin{tabular}{lllll}
\hline & $\begin{array}{l}\text { Gastric cancer } \\
(\mathbf{n}=\mathbf{2 1})\end{array}$ & $\begin{array}{l}\text { Colorectal cancer } \\
(\mathbf{n}=\mathbf{4 9})\end{array}$ & $\begin{array}{l}\text { Control } \\
(\mathbf{n = 2 0})\end{array}$ & p-value \\
\hline Irisin $(\mathbf{n g} / \mathbf{m l})$ & $33.19 \pm 11.77$ & $31.97(14.94-36.33)$ & $55.9(46.85-64.99)$ & $\begin{array}{l}<0.0001^{\mathrm{a}} \\
\end{array}$ \\
& & & & $<0.0001^{\mathrm{b}^{*}}$ \\
CEA $(\mathbf{n g} / \mathbf{m l})$ & $6.38(4.56-14.0)$ & $6.63(4.83-12.11)$ & $1.25(1.06-1.52)$ & $<0.0001^{\mathrm{a} *}$ \\
\hline
\end{tabular}

$\mathrm{p}$-value was tested by student t-test,

*p-value was tested by Mann-Whitney test

a p-value was tested difference between gastric cancer and control

b p-value was tested difference between colorectal cancers and control

Table 2. Characterization of irisin and CEA as diagnostic tool.

\begin{tabular}{ccccccc}
\hline & AUC & $\mathbf{9 5 \%} \mathbf{C I}$ & $\begin{array}{c}\text { Sensitivity } \\
\mathbf{( \% )}\end{array}$ & $\begin{array}{c}\text { Specificity } \\
\mathbf{( \% )}\end{array}$ & Cut-off & p-value \\
\hline $\begin{array}{c}\text { Gastric cancer } \\
\text { Irisin (ng/ml) }\end{array}$ & 0.94 & $0.8177-1.000$ & 90 & 100 & 30.2 & $<0.0001$ \\
CEA (ng/ml) & 1.00 & $1.000-1.000$ & 100 & 100 & 3.08 & $<0.0001$ \\
\hline $\begin{array}{c}\text { Colorectal cancer } \\
\text { Irisin (ng/ml) }\end{array}$ & 0.96 & $0.9155-1.000$ & 88.1 & 90.5 & 41.9 & $<0.0001$ \\
CEA (ng/ml) & 0.99 & $0.9866-1.000$ & 98 & 100 & 2.6 & $<0.0001$ \\
\hline
\end{tabular}




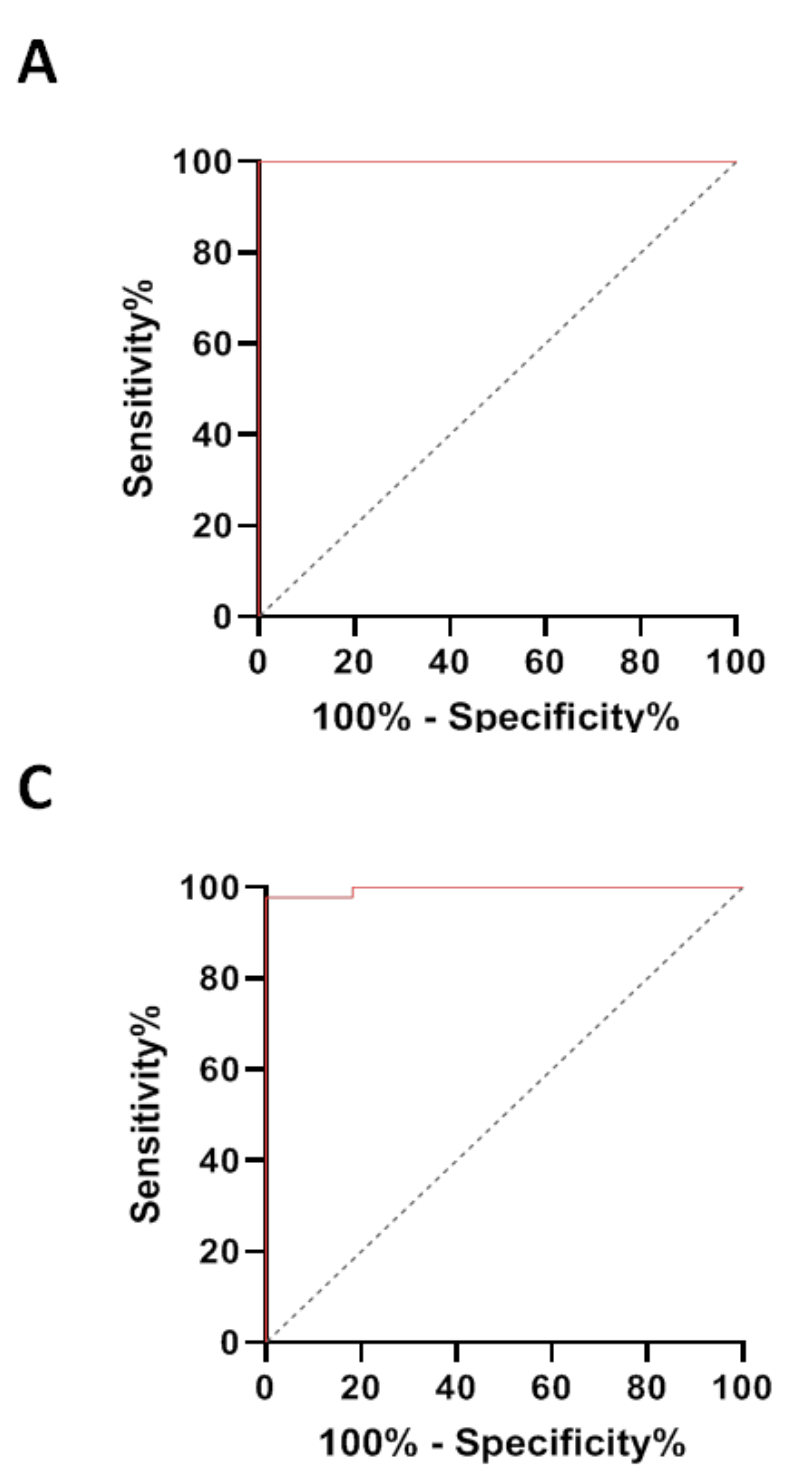

B

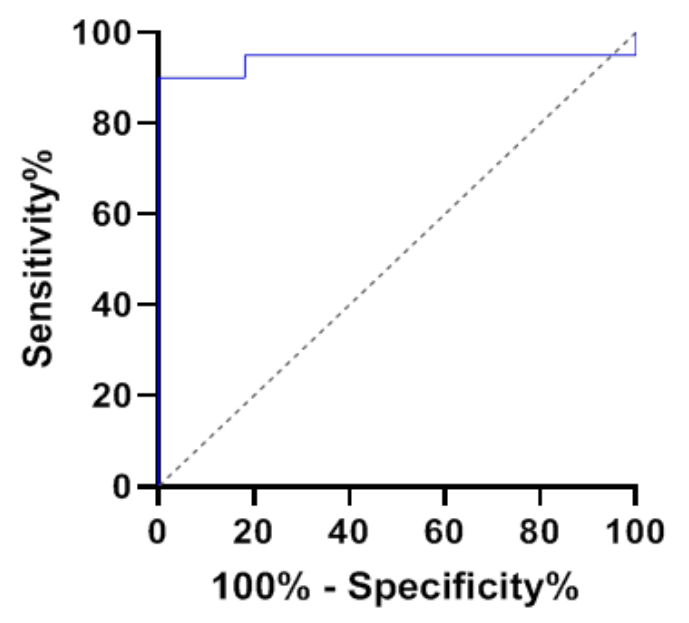

D

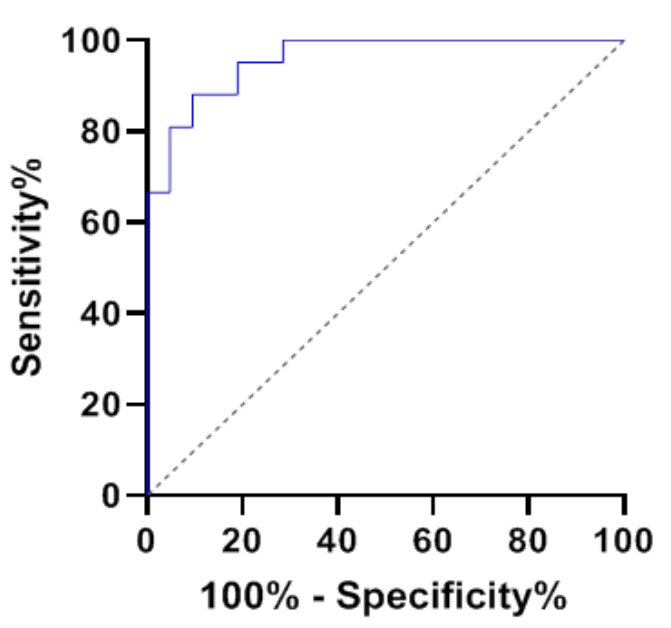

Fig.1. Receiver Operation Characteristic (ROC) Curve. A) ROC curve of CEA in Gastric cancer. B) ROC curve of irisin in gastric cancer. C) ROC curve of CEA in colorectal cancer. D) ROC curve of irisin in colorectal cancer.

\section{Discussion}

Nowadays, there have been many attempts to explore a biomarker for early detection of gastric and colorectal cancers. Several studies in different malignancies reveal the potential application of irisin as diagnostic biomarker. However, limited researches investigate diagnostic value of irisin in gastric and colorectal cancers. Therefore, the main goal of the present study is to investigate the possibility of using CEA and irisin as diagnosis biomarkers for gastric and colorectal cancer.
The most common biomarker, which is used commonly for diagnosis these types of cancer, is CEA. In current study, serum CEA level is significantly higher in gastric and colorectal cancer than in controls. This result confirm previous studies (24).

Several study in gastrointestinal cancer tissues, it was found that irisin expression increase significantly (18). Additionally, Shahidi et. al., reported that serum irisin level increase in gastric cancer patients in comparison with healthy individuals (25). In 
contrary, our study found that there is a significant decrease in irisin level in gastric cancer patients in compared to control group. This disagreement could be explained by different characteristic of patients in Shahidi's study in compared to our study. For example, in Shahidi's study, there was significant difference in BMI between gastric cancer patients and control group, while in our study; there is no significant difference in BMI

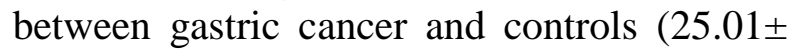
5.18 vs $26.95 \pm 3.34, p=0.054)$, respectively. Furthermore, in the present study, irisin level is significantly lower in colorectal cancer patients than in controls. In line with this result, Zhu H, et al., (2018) found that serum irisin level decrease by $23.3 \%$ in colorectal cancer patients in compared to control. Moreover, it was reported that elevated irisin reduce the risk of colorectal cancer by $78 \%$ in patients who have higher serum irisin (26). Also, in breast cancer cell lines study, it was shown that irisin reduce number of MCF-7 cells and its viability, suggesting a potential protective role of irisin from cancer (27). In the current study, reduce irisin level in cancer patients could be led to absence the protective mechanism of irisin against cancer. Therefore, further investigations are required to prove this mechanism.

Interestingly, the optimum diagnostic value of CEA and irisin were evaluated by ROC analysis. The result showed that serum CEA and irisin could differentiate gastric and colorectal cancer patients from healthy individuals. These results seem to be promising to use CEA and irisin as diagnostic biomarkers. In terms to compare between CEA and irisin, it appears that CEA is the

\section{References}

1. Sung H, Ferlay J, Siegel RL, Laversanne M, Soerjomataram I, Jemal A, et al. Global Cancer Statistics 2020: GLOBOCAN Estimates of Incidence and Mortality Worldwide for 36 Cancers in 185 Countries. CA Cancer J Clin. 2021;71(3):209-249.

2. ShaoY, Sun X, He Y, Liu C, Liu H. Elevated Levels of Serum Tumor Markers CEA and most effective biomarker to diagnose gastric and colorectal cancers than irisin. Nevertheless, irisin is still having efficient diagnostic value to discriminate these cancers patients from healthy individuals. This is the first study that compare between CEA and irisin as biomarker in gastric and colorectal cancers.

There are some factors that limit the present study; one of them is small sample size and the other did not include the measurement of other gastrointestinal cancers biomarkers such as CA19-9. Even though these limitations which were illustrated, this study reveals promising findings that could help further in early detection of gastric and colorectal cancers.

Serum CEA increase significantly and serum irisin decrease significantly in gastric and colorectal cancers. Reduce irisin level might lead to absence a potential protective mechanism of irisin against cancer. Importantly, CEA and irisin could be powerful potential diagnostic biomarkers which would be use for early detection of gastric and colorectal cancers.

Serum CEA increase significantly and serum irisin decrease significantly in gastric and colorectal cancers. Reduce irisin level might lead to absence a potential protective mechanism of irisin against cancer. Importantly, CEA and irisin could be powerful potential diagnostic biomarkers which would be use for early detection of gastric and colorectal cancers.

\section{Acknowledgements}

The authors appreciate and thank all patients for their contributions in the study.

No conflict interest is in the present study.

CA15-3 Are Prognostic Parameters for Different Molecular Subtypes of Breast Cancer. PloS one. 2015;10(7): e0133830.

3. Rashid FA, Mahdi S, Mahdy SM, Salim AT. Effect of Obesity on Plasma Alkaline Phosphatase Activity in Breast Cancer. Reports of Biochemistry and Molecular Biology. 2021;10(2):307-313. 
4. Park YA, Kang YL, Nam KK, Seung HB, Seung KS, Chang WC. Prognostic effect of perioperative change of serum carcinoembryonic antigen level: a useful tool for detection of systemic recurrence in rectal cancer. Ann Surg Oncol. 2006;13(5):645-50.

5. Huh JW, Oh BR, Kim HR, Kim UJ. Preoperative carcinoembryonic antigen level as an independent prognostic factor in potentially curative colon cancer. J Surg Oncol. 2010;101(5):396-400.

6. Shen M, Wang H, Wei K, Zhang J, You C. Five common tumor biomarkers and CEA for diagnosing early gastric cancer: A protocol for a network meta-analysis of diagnostic test accuracy. Medicine (Baltimore). 2018;97(19):e0577.

7. Boström $\mathrm{P}, \mathrm{Wu} \mathrm{J}$, Jedrychowski MP, Korde A, Ye L, Lo JC, et al. A PGC1- $\alpha$-dependent myokine that drives brown-fat-like development of white fat and thermogenesis. Nature. 2012;481(7382):463-8.

8. Roca-Rivada A, Castelao C, Senin LL, Landrove $\mathrm{MO}$, Baltar $\mathrm{J}$, Crujeiras $\mathrm{AB}$, et al. FNDC5/irisin is not only a myokine but also an adipokine. PLoS One. 2013;8(4):e60563.

9. Wen MS, Wang C, Lin S, Hung K. Decrease in irisin in patients with chronic kidney disease. PLoS One. 2013;8(5):e64025.

10. Rashid FA, Abbas HJ, Naser NA, Ali HA. Effect of Long-Term Moderate Physical Exercise on Irisin between Normal Weight and Obese Men. Scientific World Journal. 2020;2020:1897027.

11. Shelbaya S, Shady MMA, Nasr MS, Behket MM, Abd Al-Mageed Y, Abbas M. Study of Irisin Hormone Level in Type 2 Diabetic Patients and Patients with Diabetic Nephropathy. Curr Diabetes Rev. 2018;14(5):481-486.

12. Osella AR, Colaianni G, Correale M, Pesole PL, Bruno I, Buongiorno C, et al. Irisin Serum Levels in Metabolic Syndrome Patients Treated with Three Different Diets: A Post-Hoc Analysis from a Randomized Controlled Clinical Trial. Nutrients. 2018;10(7):844.

13. Park HK, Zaichenko L, Brinkoetter M, SahinEfe A, Joung KE, Tsoukas MA, et al. Circulating Irisin in Relation to Insulin Resistance and the
Metabolic Syndrome. J Clin Endocrinol Metab. 2013; 98(12):4899-4907.

14. Yan B, Shi X, Zhang H, Pan L, Ma Z, Liu S, et al. Association of Serum Irisin with Metabolic Syndrome in Obese Chinese Adults. PLOS ONE. 2014;9(4):e94235.

15. Rashid FA, Alhashemi WKH, Al- Saffar AT, Salman EA, Najeeb DA. Obesity and Breast Cancer Risk in Different Menopausal Status: A Review. Biochemical and cellular Archives. 20(1):1875-1878

16. Roomi AB, Nori W, Hamed RM. Lower Serum Irisin Levels Are Associated with Increased Osteoporosis and Oxidative Stress in Postmenopausal. Rep Biochem Mol Biol. 2021;10(1):13-19.

17. Zhang D, Tan X, Tang N, Huang F, Chen Z, Shi G. Review of Research on the Role of Irisin in Tumors. Onco Targets Ther. 2020;13:44234430.

18. Aydin S, Kuloglu T, Ozercan MR, Albayrak S, Aydin S, Bakal U. Irisin immunohistochemistry in gastrointestinal system cancers. Biotech Histochem. 2016;91(4):242-50. 19. Shao L, Lin H, Chen J, Song H, Zhang Y, We $\mathrm{F}$, et al. Irisin suppresses the migration, proliferation, and invasion of lung cancer cells via inhibition of epithelial-to-mesenchymal transition. Biochem Biophys Res Commun. 2017;485(3):598-605.

20. Liu J, Song N, Huang Y, Chen Y. Irisin inhibits pancreatic cancer cell growth via the AMPK-mTOR pathway. Sci Rep. 2018;8(1):15247.

21. Aslan R, Alp HH, Eryilmaz R, Huyut Z, Sevim M, Araz Ş, et al. Can the Irisin be a Biomarker for Prostate Cancer?. A Case Control Study. Asian Pac J Cancer Prev. 2020;21(2):505509.

22. Provatopoulou X, Georgiou GP, Kalogera E, Kalles V, Matiatou MA, Papapanagiotou I, et al. Serum irisin levels are lower in patients with breast cancer: association with disease diagnosis and tumor characteristics. BMC Cancer. 2015;15(1):898.

23. Altay DU, Keha EE, Karagüzel E, Menteşe A, Yaman SO, Alver A. The Diagnostic Value of FNDC5/Irisin in Renal Cell Cancer. Int Braz J Urol. 2018;44(4):734-739. 
24. Dolscheid-Pommerich RC, Manekller S, Walgenbach-Brünagel GK, Kalff CJ, Hartmann G, Wagner BS, et al. Clinical Performance of CEA, CA19-9, CA15-3, CA125 and AFP in Gastrointestinal Cancer Using LOCI $^{\mathrm{TM}}$-based Assays. Anticancer Research. 2017;37(1):353359.

25. Shahidi S, Hejazi J, Moghimi M, Borji S, Zabihian S, Fathi M, et al. Circulating Irisin Levels and Redox Status Markers in Patients with Gastric Cancer: A Case-Control Study. Asian Pac J Cancer Prev. 2020;21(10):2847-2851.
26. Zhu H, Liu M, Zhang N, Pan H, Lin G, Li N, et al. Serum and Adipose Tissue mRNA Levels of ATF3 and FNDC5/Irisin in Colorectal Cancer Patients with or without Obesity. Front Physiol. 2018;9:1125-1125.

27. Gannon NP, Vanghan RA, Garcia-Smith R, Bisoffi M, Trujillo KA. Effects of the exerciseinducible myokine irisin on malignant and nonmalignant breast epithelial cell behaviour in vitro. Int J Cancer. 2015;136(4):E197-202. 